\title{
Flow Structures Downstream of Square-shaped Cylinder in Channel
}

\author{
Nazım KURTULMUŞ ${ }^{1}$ \\ ${ }^{1}$ Adana Alparslan Türkeş Science and Technology University, Faculty of Engineering, \\ Department of Mechanical Engineering, Adana
}

Geliş tarihi: 01.06.2021 Kabul tarihi: 13.09.2021

\begin{abstract}
Experiments were conducted to investigate the flow topology downstream of a square-shaped cylinder situated in a straight channel. The critical parameters such as the Reynolds number $(\operatorname{Re}=500)$ and the blockage ratio of the square-shaped cylinder $(25 \%)$ were determined in light of the literature. A Particle image velocimetry (PIV) system was used to obtain the instantaneous velocity vector map. The flow structures, including the time-mean vorticity distribution, $\langle\omega\rangle$ instantaneous and time-mean streamline topology, $\Psi$, distributions of streamwise velocity fluctuations, $u_{r m s}$, turbulent kinetic energy, TKE are presented with Figures.
\end{abstract}

Keywords: Confined cylinder, Flow structures, PIV

\section{Düz Kanal İçerisine Yerleştirilmiş Kare Şekilli Silindir Ardındaki Akış Yapısı}

\section{$\ddot{O} z$}

Bu çalışmada, düz kanal içerisine yerleştirilmiş kare silindir ardındaki akış yapısını araştırma amacıyla deneyler gerçekleştirilmiştir. Çalışmadaki belirleyici parametreler, Reynolds sayısı, $R e$ ve bloklama oranı, $\beta$ sırasıyla 500 ve \%25 olarak literatür doğrultusunda belirlenmiştir. Çalışmada, anlık hız alanını ölçmek için, parçacık görüntülemeli hız ölçüm sistemi (PIV) sistemi kullanılmıştır. Zaman ortalama eşdeğer girdaplar, $\langle\omega\rangle$, ortalama ve anlık akım çizgileri, $\Psi$, akım yönündeki hız çalkantılarının karelerinin karekökü, $u_{r m s}$, türbülans kinetik enerji, TKE dağılımını içeren akış yapıları şekillerle sunulmuştur.

Anahtar Kelimeler: Akış yapıları, Sınırlandırılmış silindir, PIV

\footnotetext{
"Sorumlu yazar (Corresponding author): Nazım KURTULMUŞ, nkurtulmus@atu.edu.tr
} 


\section{INTRODUCTION}

The flow structure around bluff bodies exposed to free stream flow has attracted the attention of researchers. Studies considering the flow pattern of the square-shaped cylinder are relatively limited compared to the studies considering the flow pattern of the circular cylinder in the literature $[1,2]$. Yoon et al. [3] investigated the flow pattern of a square-shaped cylinder with different angles of incidence in laminar flow regime. Jiang and Cheng [4] investigated flow separation around a square-shaped cylinder. Zafar and Alam [5] heat transfer and flow characteristics of cylinders numerically.

Limited studies are examining the flow structure for confined cylinders. Davis et al. [6] performed on the flow pattern of a rectangular-shaped cylinder positioned in a straight channel. They investigated the effect of the inlet velocity profile, the blockage ratio, and the aspect ratio of the rectangular-shaped cylinder on the flow characteristic for the Reynolds number range of 100-1850. The investigated parameters have important consequences on the flow pattern of the rectangular-shaped cylinder. Their results showed that increasing blockage ratio causes the Strouhal number, $S t$ to increase. Camarri and Giannetti [7] performed a numerical study to investigate the flow field around a confined square-shaped cylinder. One of the characterizations of confinement, blockage ratio, $\beta$ varies between $1 / 10$ and $1 / 6$ in their work. According to them, the main characteristics that affect the inversion phenomena are the confinement, the vorticity created by the walls, and the incoming flow velocity profile. Rahnama and Hadi-Moghaddam [8] investigated the heat transfer rate of a squareshaped cylinder in a channel numerically. The working fluid and the blockage ratio, $\beta$ was determined as air and $1 / 8$, respectively. Their results revealed that the vortex shedding begins at Reynolds number, $\mathrm{Re}=60$ and steady flow characteristics continue up to $\operatorname{Re}=50$. The Nusselt number, $N u$ increases with increasing Reynolds number, Re. Sharma and Eswaran [9] conducted a numerical analysis to investigate the flow patterns and heat transfer rates of a confined and unconfined square-shaped cylinder. The blockage ratio varies from $\% 0$ (unconfined case) to $\% 50$ and the Prandtl number, $\mathrm{Pr}$ is chosen as 0.7. Their results showed that the drag coefficient of the square-shaped cylinder raises with a rising blockage ratio, $\beta$. The increment of the strength of vortices from the square-shaped cylinder and the upgraded entrainment between the fluid close to the channel wall and the fluid close to the squareshaped cylinder provides enhancement of Nusselt number, $N u$ of the cylinder with rising blockage ratio, $\beta$ except for $20 \%$. Reyes et al. [10] used Particle Image Velocimetry (PIV) system to investigate qualitative flow characteristics of a square-shaped cylinder located in parallel plates. They also used numerical methods to compare the results obtained from the experimental method. The blockage ratio is determined as $1 / 2.5$ and the Reynolds number, $R e$ varies from 100 to 256 . They observed three different flow regimes: a steady recirculating bubble regime, an unsteady recirculating bubble regime, and a Karman vortex street flow regime. Athinarayanan et al. [11] performed a numerical analysis to examine the heat transfer rate of a square-shaped cylinder positioned in a channel. The blockage ratio, $\beta$ is determined as $25 \%$ and the working fluid is $\mathrm{Cu}-$ water nanofluid. The Reynolds number, $R e$ varies from 10 to 40 and the volume fraction of nanofluid varies from 0 to 0.1 . According to them, introduced nanoparticles compared with the base fluid play an important role in enhancing heat transfer. Dhiman et al. [12] studied the effect of Peclet number, $P e$ on flow pattern and heat transfer rates of a square-shaped cylinder positioned in a channel in the steady flow regime. Prandtl number, $P r$ varies from 0.7 to 4000 . The effect of blockage was also investigated. They showed that the average Nusselt number, $\mathrm{Nu}$ upgrades with rising the Reynolds number and Prandtl number, $P r$. Their further investigation aims to investigate the effects of power-law index and blockage ratio on the flow pattern of a confined square-shaped cylinder [13]. Hegedüs et al. [14] conducted some measurements to investigate the flow parameters on the rectangularshaped cylinder in the channel. The rectangularshaped cylinders had four different aspect ratios and the blockage ratio was chosen as $12.5 \%$. 
According to them, a slight increase of the Strouhal number, $S t$ is observed with an increasing aspect ratio. Shadaram et al. [15] conducted experiments to obtain flow data on a rectangularshaped cylinder positioned in a straight channel using hot-wire anemometer device. The effect of the height-width ratio of the cylinder on flow data was investigated. With an increasing height-width ratio of the rectangular-shaped cylinder, the turbulence intensity in wake flow decreases. Farhadi and Rahnema [16] performed a numerical analysis to investigate flow parameters of a squareshaped cylinder positioned in a straight channel for Reynolds numbers, $R e$ such as $22 \times 10^{3}$ and $21.4 \times 10^{3}$. Kim et al. [17] used a large eddy simulation method to examine the flow pattern of square-shaped cylinders in a channel. The blockage ratio, $\beta$ was implemented as $20 \%$ in their work and the Reynolds number is fixed at 3000 . A uniform velocity profile is imposed as an inlet boundary condition. According to them, the channel wall has a remarkable effect on the flow parameters of a square-shaped cylinder such as higher turbulent fluctuation compared to an unconfined one. Channel walls reduce the circulating region size in the wake behind the cylinder. Additionally, the drag force values increase. Nakagawa et al. [18] conducted experimental studies on a rectangular-shaped cylinder's heat transfer and flow pattern positioned in a straight channel. The ratio between width and height of the rectangular-shaped cylinder varies such as $0.5,1,2,3$. They concluded that the heat transfer enhancement is caused by the vortices shedding from the rectangular-shaped cylinder. Nakagawa et al. [19] used a Laser Doppler Velocimetry system to examine the flow pattern of a rectangular-shaped cylinder positioned in a straight channel. They applied the blockage ratio as $20 \%$ and uniform velocity inlet conditions in their experiments. According to them, the turbulent intensities at the middle height of the straight channel get a maximum value close to the stagnation point of a recirculation region. The ratio between width and height of the rectangularshaped cylinder substantiallly affects on the coherent flow structures. Ortega-Casanova [20] conducted a numerical analysis to investigate the effect of aspect ratio on flow characteristics of a rectangular-shaped cylinder in a channel. The blockage ratio was determined as $\% 20$ and the aspect ratio (width-height ratio) varies between $1 / 8$ and 4. According to them, the lowest critical Reynolds number is obtained as 30 for the aspect ratio of $1 / 8$. The Strouhal number, St is 0.23 for aspect ratio $\leq 1$ and 0.253 for aspect ratio $\geq 2$. Rosales et al. [21] studied heat transfer rates and flow patterns of a square-shaped cylinder positioned in a straight channel numerically. They investigated the wall effect on the vortex shedding from the cylinder and the effect of the upstream eddy promoter on the flow pattern and heat transfer rates on the cylinder. The simulations were performed at $\mathrm{Re}=500$. Their results showed that the eddy promoter enhances the heat transfer rate and decreases the drag from the downstream cylinder. The vortex shedding from the cylinder close the wall structure different from the vortex shedding from the cylinder located symmetrically in the channel.

The best of the author's knowledge, there is no work on the flow pattern of a square-shaped cylinder located in a straight channel by using the Particle image velocimetry (PIV) system. The flow patterns, including the time-mean vorticity distribution, $\langle\omega\rangle$ instantaneous and time-mean streamline topology, $\Psi$, distributions of streamwise velocity fluctuations, $u_{r m s}$, turbulent kinetic energy, $T K E$ are investigated.

\section{MATERIALS AND METHODS}

\subsection{Experimental Set-Up}

The same experimental set-up introduced by Kurtulmus et al. [22] were used to perform the experiments to examine the flow pattern of the square-shaped cylinder. However, it is necessary to explain the details of the set up briefly.

A pump with a frequency controller pumps the water from a water tank to the water channel. The water flow rate can be changed from $0 \mathrm{~m}^{3} / \mathrm{h}$ and $1.8 \mathrm{~m}^{3} / \mathrm{h}$. An electromagnetic flowmeter measures the volume flow rate which range of $0.17 \mathrm{~m}^{3} / \mathrm{h} \leq Q$ $\leq 17.6 \mathrm{~m}^{3} / \mathrm{h}$. The electromagnetic flowmeter was mounted to the water channel before the entrance. 


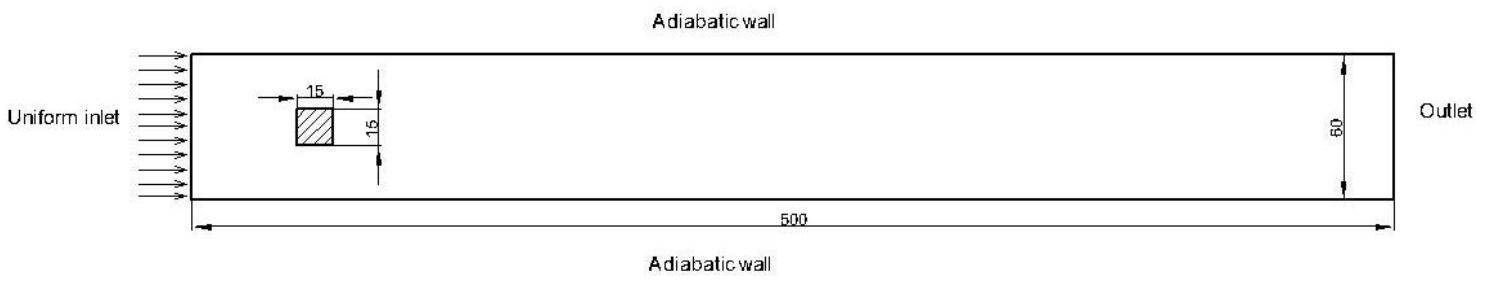

Figure 1. The model tested in the experiments the study

With the directions of the the manufacturer's catalog, pipes with $0.5 \mathrm{~m}$ and $1.5 \mathrm{~m}$ lengths were mounted to inlet and outlet sections of the electromagnetic flowmeter. The pipes have $25 \mathrm{~mm}$ inner diameter. The water enters the closed water channel after the volume flow rate is measured. The closed water channel components are flow straightener section, the diffuser section, the contraction section and the test section. $1 \mathrm{~cm}$ thick plate from Plexiglas material is used to produce the closed water channel. The flow straightener section consisted of honeycomb and screen combinations to reduce turbulence at the inlet of the test section. After the flow exits from the flow straightener section, it enters the test section passing through the contraction section. The water moves forward to the thermal regulation system. This thermal regulation system consists of a water reservoir, a heater and a chiller. The heater is coupled with the water reservoir to regulate the water temperature by means of a variable transformer. Then the water is cooled with the chiller. This system is used to keep the temperature of the water constant, which is $17{ }^{\circ} \mathrm{C}$ at the test section inlet. After all, the water returns to the water tank to recycle again.

\subsection{Measurement of Flow Characteristics}

Instantaneous velocity measurements were performed using Particle image velocimetry (PIV) system to investigate flow topology downstream of square-shaped cylinder. A laser light sheet from $\mathrm{Nd}$ :YAG laser units illuminates the test section. The laser sheet which has two mm thick was oriented at the midplane of the test section. Therefore, the sidewall effect on the flow is eliminated. $1.5 \mathrm{~ms}$ is the time interval between the laser pulses. The image capturing was accomplished with a CCD camera. Hollow glass sphere particles seeded. They had a diameter of $\sim 10 \mu \mathrm{m}$. One thoausand instantaneous images of the flow were obtained and logged to the computer for each flow characteristics experiment using the PIV system. 15 images per second can be obtained from the PIV system.

\subsection{Test Section}

The channel shown in Figure 1 is used as the test section. The test section includes the channel consisted of the side, upper and lower plates and the square-shaped cylinder placed in the channel. This channel was constructed using $1 \mathrm{~cm}$ thick plexiglass plates in order to provide laser transmission and flow visualization. In the light of the literature, the height of the channel is $60 \mathrm{~mm}$ and the length of one side of the square, $D$ was chosen to be $15 \mathrm{~mm}$ in order to provide the blockage ratio as $25 \%$ [11]. The square-shaped cylinder was placed $3.8 \mathrm{D}$ from the channel's entrance and in the middle of the channel height. To investigate flow characteristics downstream of the square-shaped cylinder, the model shown in Figure 2 were prepared.

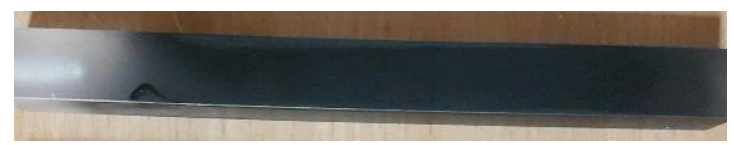

Figure 2. The model tested in the experiments

\section{RESULTS AND DISCUSSIONS}

The hydrodynamics downstream of the squareshaped cylinder in the test section is presented. The flow structures including the time-mean vorticity distribution, $\langle\omega\rangle$ instantaneous and time-mean streamline topology, $\Psi$, distributions of streamwise velocity fluctuations, $u_{r m s}$, turbulent kinetic energy, $T K E$ are presented with Figures. 


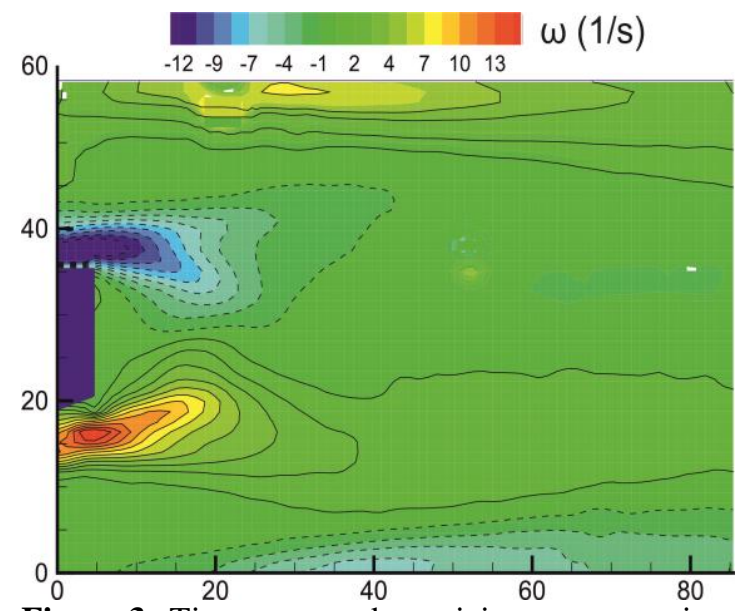

Figure 3. Time-averaged vorticity concentration, $\langle\omega\rangle$ for square-shaped cylinder

The time-mean vorticity contours, $\langle\omega\rangle$ is depicted in Figure 3 for the square-shaped cylinder located in a straight channel. Time-mean vorticity patterns, $\langle\omega\rangle$ quantity can be followed from the legend. In Figure dashed lines shows the negative vorticity patterns, $\langle\omega\rangle$ and solid lines shows the positive vorticity patterns, $\langle\omega\rangle$. The time-mean vorticity clusters, $\langle\omega\rangle$ form at the upstream edge of the square-shaped cylinder and shed through the shear layers. Entrainment between the mean flow and wake regions occurs due to the shear layer instabilities as well as vortex shedding. There are also vorticity clusters near the upper and lower wall just near downstream of the square-shaped cylinder. When one examines the instantaneous vorticity distribution through the flow, the interaction between the vortices shedding due to the existence of square-shaped cylinder and the vorticity clusters generated on the upper and lower wall boundary is clearly seen. This flow phenomenon is expected to increase heat transfer enhancement from the wall because of the disturbance of the boundary layer on the wall.

Figure 4 shows the time-mean streamlines topology, $\langle\Psi\rangle$, for the square-shaped cylinder. The counter-rotating recirculating zones are taken place just behind the square-shaped cylinder due to the flow separation at upstream corners of the square-shaped cylinder.

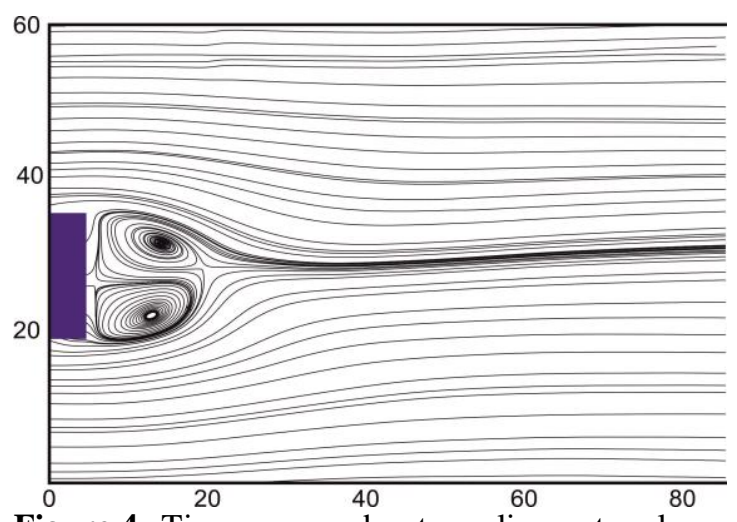

Figure 4. Time-averaged streamlines topology, $\langle\Psi\rangle$, for square-shaped cylinder

The instantaneous streamlines topology, $\Psi$ for square-shaped cylinder placed in a channel over a vortex shedding phenomena is demonstrated in Figure 5. Eight frames with the time interval of $5 \Delta \mathrm{t}$ in each one were selected to follow the flow pattern. Here, the $\Delta \mathrm{t}$ represents the time interval that each frame is obtained from the PIV system. The flow separates from the wall boundary due to the abrupt geometrical change, and forms the wake region behind the square-shaped cylinder. The wake region instability is clearly seen from the instantaneous streamlines topology. In accordance with vortex shedding phenomenon, recirculation regions at the upper edge and lower edge of the square-shaped cylinder shed alternately.

Figure 6 shows the time-mean rms maps of the $u$ velocity corresponding to $\mathrm{Re}=500$ for the squareshaped cylinder located in the straight channel. The fluctuations of streamwise velocity upgrade the heat transfer rate [23]. The distribution of $u_{r m s}$ is denser relatively through the shear layer between the main and wake recirculation flow regions.

The instability of vorticity, and the magnitude of the turbulence mixing, is represented as turbulent kinetic energy, TKE. The incremental value of $T K E$ for the square-shaped cylinder shown in Figure 7 is 0.05 . It is normalized by dividing averaged stream velocity. As shown in Figure, the high-level TKE contours intensifies behind the square-shaped cylinder where the separated shear layers from the upper and lower sides of the square-shaped cylinder merge. 

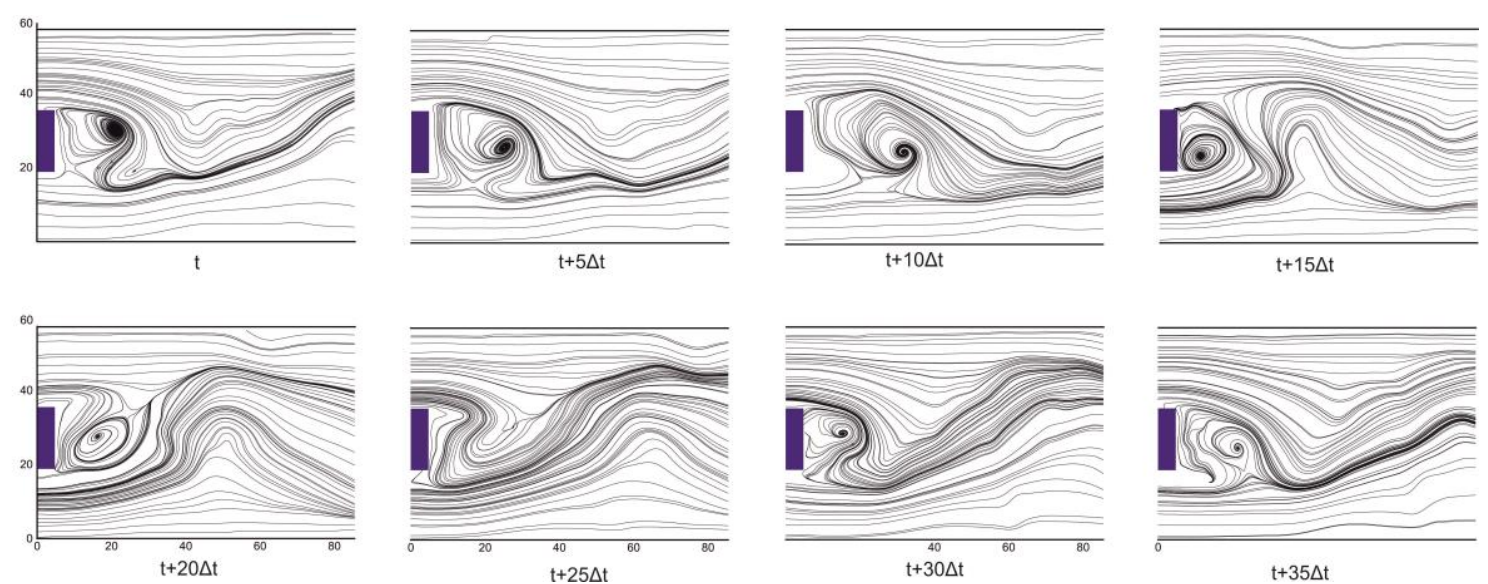

$\mathrm{t}+15 \Delta \mathrm{t}$



Figure 5. Instantaneous streamlines topology, $\Psi$ over a vortex shedding phenomena for square-shaped cylinder

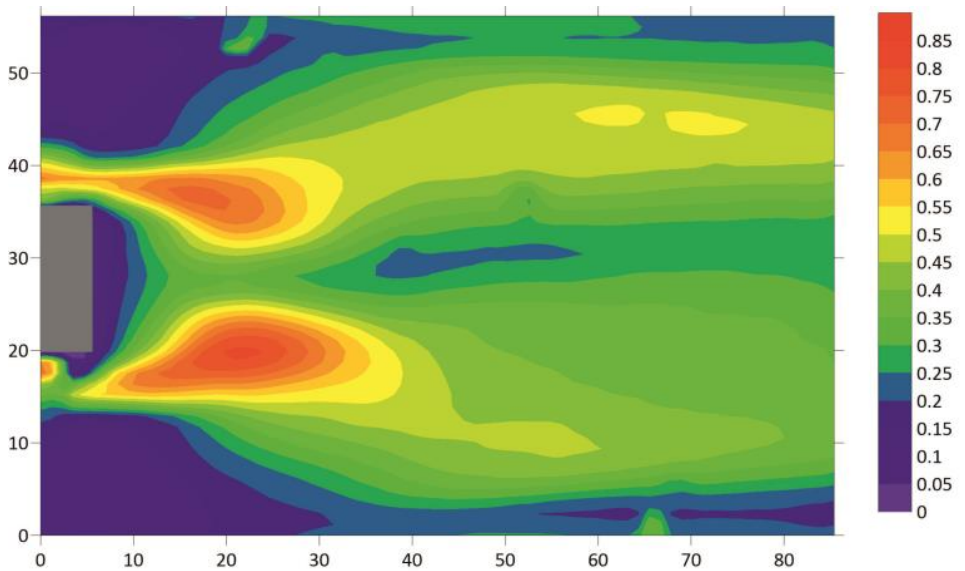

Figure 6. Distributions of streamwise velocity fluctuations for square-shaped cylinder, $u_{r m s}$



Figure 7. Patterns of turbulent kinetic energy, $T K E$ for square-shaped cylinder 


\section{CONCLUSION}

In this study, experiments were performed to investigate the flow topology downstream of a confined square-shaped cylinder. A particle image velocimetry (PIV) system was used to obtain the instantaneous velocity vector map. The results show that the flow separates at the upstream edge of the square-shaped cylinder and the wake region forms behind the cylinder. Placing a square-shaped cylinder in the channel enhances the interactions between the mean flow and the flow close the channel wall. In addition, the vortices generated from the wall and the vortices shedding from the edge of the squared cylinder encounter downstream of the flow. This flow phenomenon provides enhanced mixing flow in the channel.

\section{ACKNOWLEDGMENTS}

The author acknowledges the Mechanical Engineering Department at Cukurova University for the opportunity to perform experiments in the Fluid Mechanics Laboratory.

\section{REFERENCES}

1. Zdravkovich, M.M., 1997. Flow around Circular Cylinders, Vol. 1: Fundamentals. Oxford University Press, New York.

2. Williamson, C.H.K., 1996. Vortex Dynamics in the Cylinder Wake. Ann. Rev. Fluid Mech., 28, 477-539.

3. Yoon, D.H., Yang, K.S., Choi, C.B., 2010. Flow Past a Square-shaped Cylinder with an Angle of Incidence. Physics of Fluids, 22, 043603.

4. Jiang, H., Cheng, L., 2020. Flow Separation Around a Square-shaped Cylinder at Low to Moderate Reynolds Numbers. Physics of Fluids, 32, 044103.

5. Zafar, F., Alam, M., 2019. Flow Structure Around and Heat Transfer from Cylinders Modified from Square to Circular. Physics of Fluids, 31, 083604.

6. Davis, R.W., Moore, E.F., Purtell, L.P., 1984. A Numerical-experimental Study of Confined
Flow Around Rectangular-shaped Cylinders. Physics of Fluids, 27, 46- 59.

7. Camarri, S., Giannetti, F., 2007. On the Inversion of the von Karman Street in the Wake of a Confined Square-shaped Cylinder. Journal of Fluid Mechanics, 574, 169-178.

8. Rahnama, M., Hadi-Moghaddam, H., 2005. Numerical Investigation of Convective Heat Transfer in Unsteady Laminar Flow over a Square-shaped Cylinder in a Channel. Heat Transfer Engineering, 26, 21-29.

9. Sharma, A., Eswaran, V., 2005. Effect of Channel Confinement on the Two-dimensional Laminar Flow and Heat Transfer across a Square-shaped Cylinder. Numerical Heat Transfer, Part A, 47, 79-107.

10. Reyes, M., Velazquez, A., Martin, E., Arias, J.R., 2013. Experimental Study on the Confined 3D Laminar Flow Past a Square Prism with a High Blockage Ratio. International Journal of Heat and Fluid Flow, 44, 444-457.

11. Athinarayanan, A.S.K., Gurunathan, M., Parthasarathy, R.K., Taler, J., Oclon, P., Taler, D., 2019. Numerical Investigation of Heat Transfer from Flow Over Square-shaped Cylinder Placed in a Confined Channel Using $\mathrm{Cu}$-water Nanofluid. Thermal Science, 23, 1367-1380.

12. Dhiman, A.K., Chhabra, R.P., Eswaran, V., 2005. Flow and Heat Transfer Across a Confined Square-shaped Cylinder in the Steady Flow Regime: Effect of Peclet Number. International Journal of Heat and Mass Transfer, 48, 4598-4614.

13. Dhiman, A.K., Chhabra, R.P., Eswaran, V., 2008. Steady Flow Across a Confined Squareshaped Cylinder: Effects of Power-law Index and Blockage Ratio. J. Non-Newtonian Fluid Mech., 148, 141-150

14. Hegedűs, F., Hős, C., Pandula, Z., Kullmann, L., 2010. Measurement on the Cavitating Vortex Shedding Behind Rectangular-shaped Obstacles. IOP Conf. Ser.: Earth Environmental Science, 12, 012066.

15. Shadaram, A., Fard, M.A., Rostamy, N., 2008. Experimental Study of Near Wake Flow Behind a Rectangular-shaped Cylinder. 
American Journal of Applied Science, 5, 917-926.

16. Farhadi, M., Rahnama, M., 2005. Threedimensional Study of Separated Flow Over a Square-shaped Cylinder by Large Eddy Simulation. Proc. IMechE Part G: J. Aerospace Engineering, 219, 225-234

17. Kim, D.H., Yang, K.S., Senda, M., 2004. Large Eddy Simulation of Turbulent Flow Past a Square-shaped Cylinder Confined in a Channel. Computers \& Fluids, 33, 81-96.

18. Nakagawa, S., Senda, M., Kikkawa, S., Wakasugi, H., Hiraide, A., 1998. Heat Transfer in Channel Flow Around a Rectangular-shaped Cylinder. Heat Transfer-Japanese Research, 27, 84-97.

19. Nakagawa, S., Nitta, K., Senda, M., 1999. An Experimental Study on Unsteady Turbulent Near Wake of a Rectangular-shaped Cylinder in Channel Flow. Experiments in Fluids, 27, 284-294.

20. Ortega-Casanova, J., 2017. On the Onset of Vortex Shedding from 2D Confined Rectangular-shaped Cylinders Having Different Aspect Ratios: Application to Promote Mixing Fluids. Chemical Engineering \& Processing: Process Intensification, 120, 81-92.

21. Rosales, J.L., Ortega, A., Humphrey, L.A.C., 2000. A Numerical Investigation of the Convective Heat Transfer in Unsteady Laminar Flow Past a Single and Tandem Pair of Squareshaped cylinders in a Channel. Numerical Heat Transfer, Part A, 38:443-465.

22. Kurtulmuş, N., Zontul, H., Sahin, B., 2020. Heat Transfer and Flow Characteristics in a Sinusoidally Curved Converging-diverging Channel. International Journal of Thermal Sciences 148, 106163.

23. Chen, T.Y., Du, R.K., 2003. Effects of Velocity Fluctuations on Heat Transfer Enhancement. Experiments in Fluids, 34, 548-555. 